\title{
La participación comunal en Colombia y Venezuela ¿Privatización o Democracia Participativa?
}

Nila Leal González*

\section{Resumen}

El trabajo analiza fundamentalmente los mecanismos y organizaciones de participación comunal y vecinal, para establecer en base a la comparación de sus caracteristicas algunas semejanzas y diferencias en los instrumentos legislativos y en los resultados obtenidos en su aplicación que permiten comprender las tendencias de los procesos participativos iniciados en Colombia y Venezuela. El trabajo concluye que ambas reformas poltticas con una orientación neoliberal o privatizadora reducen a la participación comunal y vecinal en una participación-gestión que no conduce a la democratización en los sistemas politicos de estos paises.

Palabras claves: Venezuela, Colombia, Participación Comunitaria, Privatización, Democracia.

\section{The communal participation in Colombia and Venezuela ¿Privatizacion or Participative Democracy?}

\section{Asbtract}

The work fundamentally analyzes the mechanisms and organizations of communal and neighborhood participation, to establish based on the comparison of their characteristics some similarities and differences on the legislative instruments and on the results obtained in its aplication which help understand the tendencies of the participative

Recibido: 09-01-96 , Aceptado: 25-03-96

- Protesora titular e investigadora del Departamento de Ciencias Humanas de la Facultad Experimental de Clenclas, adscrita al instituto de Estudios Politicos y Derecho Público. Universidad del Zulia. Maracalbo-Venezuéla. 
processes initiated in Colombia and Venezuela The work concludes that both political reforms with a neoliberal or a privatization orientation reduce the communal and neighborhood participation in a action - participation that is non-conducive to democratization in the political system of these countries.

Key words: Venezuela, Colombia, Community Participation, Privatization, Democracy.

\section{Introducción}

En Colombia y Venezuela, debido al proceso de complejización creciente de sus sociedades y a los procesos que se están dando condicionados por la crisis mundial; regional y nacional; las instituciones y mecanismos de participación y representación política dejaron de adecuarse a esta nueva situación sociopolitica por escasos, inoperantes y excluyentes.

En consecuencia, se impuso la necesidad de abrir nuevos espacios politicos cuya expresión se encontraba limitada por los sistemas institucionales vigentes y las prácticas que de ellos se derivaban: clientelisismo, cooptación o mediatización partidista, concentración de poder.

Las exigencias planteadas por la actual dinámica política en crisis, imponian consecuentemente la necesidad de diversificar los canales y mecanismos de participación, y de mejorar la calidad de la representación a fin de incrementar la acción y expresión autónoma de los intereses de los distintos actores que integran los sistemas políticos.

Consiguientemente, una mayor diversificación de los centros de gravedad de la democracia y de sus instituciones y organizaciones constitutivas debería tender a la distribución más equitativa de los recursos politicos, sociales y económi- $\cos$, lo que permitirla la redemocratización o la posibilidad de una democracia más participativa donde la mayoria de los ciudadanos puedan intervenir en el proceso de toma de decisión sobre los asuntos que conciernan a su vida. La participación entonces implicaria; no sólo la ampliación cuantitativa de mecanismos, instancias y actores, sino fundamentalmente la recomposición cualitativa que además de lo político; abarque lo social y lo económico, lo que supone una nueva rearticulación Estado-Sociedad Civil.

En la praxis, la participación ciudadana y comunitaria deberian traducirse en una participación política o decisional en la selección de los gobernantes y directa 0 indirectamente en la formulación de las políticas públicas. Esta participación, conjuga la representación con la participación directa o la intervención efectiva de los ciudadanos en la toma de decisiones.

No obstante, este proceso democratizador, puede ser desviado hacia otras orientaciones, la tendencia neoliberal propicia una democracia que promueve la reducción o limitación de la actividad del Estado en la vida económica, social y cultural de la sociedad civil, esto implica el traslado de algunas funciones que el Estado habia venido ejerciendo hacia la estera social o la privatización de las poÍticas públicas. Esta tendencia propone 
una participación ciudadana y comunitana que se limita a la gestión sin incidencia decisional (es fundamentalmente una participación representativa), y el reacomodo de actores, mecanismos e instituciones para mantener la cooptación o mediatización de las organizaciones ciudadanas y la concentración de poder.

A mediados de la década de los 80 , Colombia y Venezuela inician procesos de reforma politica enmarcados en la necesidad de buscar una nueva interrelación Estado-Sociedad Civil, para extender el poder que hasta ahora habla sido ocupado casi exclusivamente por la socledad política, al campo de la sociedad civil en sus diversas articulaciones, organizaciones, gremios y asociaciones. Esto implicaba por un lado, los procesos de descentralización polftico-administrativa del Estado y por otro, la promoción y estimulo de la participación ciudadana a través de la creación de una serie de instancias y mecanismos politicos y sociales y de organizaciones comunitarias o comunales; entre otras asociaciones.

En este trabajo se analizarán fundamentalmente los mecanismos y organizaciones de participación comunal de Colombia y Venezuela (Juntas de Acción Comunal de Colombia y Asociaciones de Vecinos de Venezuela), las cuales están vinculadas a las necesidades inmediatas de las comunidades, principalmente urbanas, para establecer en base a la comparación de sus características, algunas semejanzas y diferencias en los instrumentos legislativos y en los resultados obtenidos en su aplicación; las tendencias de los procesos participativos iniciados en ambos paises.

\section{Los Instrumentos}

Para poner en marcha las reformas politicas e impulsar la participación ciudadana y comunitaria fue necesario promulgar una serie de leyes y reglamentos que incentivaran y regularan estos procesos.

En Colombia, la Constitución Política de 1991 (vigente actualmente) asume como elemento definitorio del Estado la participación ciudadana y comunitaria (art. 1), a partir de la incorporación de la participación como principio constitucional, es obligación del Estado, defenderla y promoverla en todas las actividades de la vida pública (art. 2). Así mismo, la participación del pueblo en las decisiones se convierte en definición de la soberanía, al establecer que el pueblo la ejercerá en forma directa o por medio de sus representantes (art. 3). A partir de alli, la Constitución Colombiana reconoce múltiples derechos de participación económica, poIftica y social, cultural y territorial y establece similares mecanismos instancias y obligaciones encaminadas a la promoción de la participación ciudadana y comunitaria en las politicas públicas y de interés común. Participación ésta, que es definida como un derecho y un deber del ciudadano.

La Carta Colombiana de 1991, sienta las bases para el tránsito de la democracia representativa hacia la democracia participativa. De esta manera se pretende generar un nuevo modelo de organización de la sociedad, en la cual la participación como principio organizativo por excelencia, se constituye en el mecanismo de articulación de las relaciones 
sociales, del Estado con los ciudadanos $y$ de estos entre sl.

Para lograr esto se establecen tres maneras de modificar las relaciones Estado-Sociedad Civil a través de la participación: de gestión directa de la administración pública (socialización del Estado); cogestión de los servicios públicos, equiparando las responsabilidades jurídicas del Estado con las de la sociedad en materia de polfticas públicas; $y$ de privatizar la cosa pública o delegar sobre los ciudadanos la propiedad o responsabilidad de lo público (desmantelamiento del Estado).

Esto supone que la democracia participativa y las modificaciones que introduce en las relaciones entre el Estado y la sociedad deberán abarcar lo político y lo social e implicarán distinguir múltiples actores: individuales (ciudadanos), colectivos (comunidad) y asociativos (gremio) (Ochoa y Restrepo, 1993).

No obstante, el ordenamiento juridico de la participación contenido en el texto constitucional, desarrolla principalmente la participación política o decisional, es decir, los mecanismos necesarios para ejercer el poderpolitico y profundizar la relación de la ciudadanía con el Estado. Estos mecanismos son: el voto, plebiscito, referendo, consulta popular, cabildo abierto, iniciativa legislativa y revocatoria del mandato ${ }^{1}$.

En forma más débil, la Constitución, se refiere a la participación social o de gestión, esto es a los mecanismos requeridos por la organización comunita- ria para actuar en función de la solución de problemas colectivos y en la promoción del bien común (en el orden político y social), como también, para canalizar los intereses particulares y poder asi relacionarse con otros grupos y con el Estado mismo (Ochoa y Restrepo, 1993). Sin embargo, en base a esta Carta Constitucional, se han promulgado la Ley de Participación y la Ley Modernización y Funcionamiento de los Municipios, que junto con un conjunto de Decretos normatizan el funcionamiento de la organización comunitaria. En Venezuela la Constitución del 1961 (vigente actualmente), sólo consagra la democracia representativa (Art. $3^{\text {p}}$ ). La participación ciudadana ha sido concebida en nuestra legislación desde una perspectiva electoral, es decir en términos de la selección de nuestros gobernantes (derecho de elegir) y de la postulación a los cargos (derecho a ser elegido), dejando en un plano marginal la expresión de la participación a través de la influencia que la comunidad pueda ejercer en la toma de decisión y en la elaboración de las políticas públicas. La participación decisión se establece en el texto constitucional en forma indirecta a desarrollarse con la mediación de los partidos politicos.

Salvo las disposiciones contenidas en los Artículos 246 y 165 ejusdem, relativos a la consulta popular directa y obligatoria en sus efectos, en el caso de reforma a la Constitución, de la iniciativa popular de las leyes y de la posibilidad de establecer organismos de carácter con-

1 Ver en la Constitución Colombiana (1991) los Artículos 40, 104, 105, 106, 155, 170, 259, 375 y 378. 
sultivo con la participación de los particulares; los mecanismos anteriormente sehalados son los fundamentales en el texto constitucional para la incidencia de los ciudadanos en la conducción politica de la vida nacional (Alvarez y Morales, 1993:131). Sin embargo, no se observan en la Constitución de 1961 razones de tipo legal que impidan a través de la promulgación de algunas leyes consagrar otros medios de participación.

De all que, con la reforma polftica que se adelanta en Venezuela a partir de 1989, se promulga la Ley Orgánica de Régimen Municipal la cual establece una serie de mecanismos de participación poIftica como la iniciativa legislativa local, el recurso de referéndum, la solicitud de reconsideración de ordenanzas y la revocatoria del Mandato del Alcalde. Además pueden mencionarse los Cabildos Abiertos, la pérdida de investidura de alcaldes $y$ concejales en el control patrimonial de los bienes municipales y la iniciativa popular sobre creación de entidades loca* les ${ }^{2}$. La Legislación Municipal también establece el Reglamento para la participación-gestión por medio de las Asociaciones de Vecinos como expresión organizada de las comunidades y la posibilidad de constituir otras formas organizativas comunitarias, se establecen mecanismos de participación a través de los cuales, la comunidad puede incidir en la gestión de los servicios públicos locales. A tal efecto se han creado una serie de instancias en esta legislación para viabilizar estos mecanismos. No obstante, es- tos mecanismos siguen siendo insuficientes y limitados. Asi mismo, a nivel de los Estados regionales y a nivel nacional hacen falta urgentes mecanismos de participación.

A diferencia de la legislación venezolana, la colombiana con un excesivo énfasis tecnocrático presenta un marco jurídico-constitucional más amplio y definido, estableciendo multiplicidad de derechos, instancias y mecanismos que posibilitan el ejercicio de la participación ciudadana. A pesar de este mayor desarrollo legislativo los mecanismos igualmente presentan limitaciones en su conceptualización y en consecuencia en su aplicación. Es importante señalar, que en ambas reformas la participación ciudadana es considerada un elemento esencial de la búsqueda de la nueva relación EstadoSociedad Civil. Además en ambas legislaciones (con una definición más precisa en la legislación colombiana), se diferencian los mecanismos de participación poIftica de los mecanismos de participación social.

Interesa en el análisis que se realiza; ahondar sobre la participación social - participación-gestión, puesto que sin dejar de reconocer la importancia y complementariedad de los mecanismos políticos, la participación-gestión está estrechamente vinculada a las necesidades más inmediatas de la comunidad que dia a dia deben enfrentar diversos problemas (viviendas, servicios públicos, seguridad personal) y que difícilmente podrán resolver a través de mecanismos tan compliy 179. 
cados en su operativización como los mecanismos de participación politica ${ }^{3}$, que además sólo pueden utilizarse para determinadas situaciones y no para la sobrevivencia cotidiana.

\section{Legislación Comunal y Vecinal}

La acción comunal y vecinal aparece incorporada en los dos sistemas pollticos a partir de 1958, esto es, forman parte de las reformas institucionales $y$ politicas que el Frente Nacional en Colombia y el Pacto de Punto Fijo en Venezuela establecen en aras de modernizar sus respectivas estructuras institucionales. Los objetivos formales señalaban la importancia de promover la organización y participación de las comunidades locales para facilitar el mejoramiento de sus condiciones de vida y su acceso al desarrollo.

Los programas para la organización y participación de las comunidades en estos dos palses, incluso con apoyo internacional ${ }^{4}$ fueron programas concretos dirigidos institucionalmente desde ese momento.

Además desde el comienzo la organización comunal y vecinal, tiene en Colombia y Venezuela un carácter ambiguo; al mismo tiempo que son formas de organización que se aspira sean autónomas, dependen de las regulaciones, apoyo y manejo de las instituciones estatales.
Tal condición ambigua les acompaña en su posterior evolución.

La actividad de estas organizaciones se ha desarrollado en el ámbito de lo local, en el entorno más próximo de las comunidades. De all, que su papel y actividad se relaciona principalmente con los Concejos Municipales quienes deben responder a las demandas de las comunidades, al mismo tiempo que de regular y supervisar las organizaciones vecinales.

El desarrollo de la organización comunal y vecinal y su inserción definitiva en los sistemas socio-políticos de ambos países se produjo dentro de un proceso que tuvo varias etapas que pueden observarse al definir los cambios legislativos de las Juntas de Acción Comunal en Colombia y las Asociaciones de Vecinos en Venezuela, en los organismos encargados de su regulación y en el propio desarrollo de las organizaciones vecinales.

Esta evolución se ha dado a través de un proceso que comprende por una lado las exigencias y demandas de las comunidades que se organizaban y por otro lado, la búsqueda de los Estados y sistemas políticos por institucionalizar y adecuar mecanismos para mantener bajo control tales organizaciones a través de las regulaciones homogéneas (leyes $y$ reglamentos) en todo el territorio nacional, asegurando de esta manera su poder político. No obstante, la aparición y conti-

3 Ochoa y Restrepo (1993) hacen un análisis de las instituciones y los mecanlsmos de participación ciudadana, señalando las limitaciones que presentan los mecanismos de participación política: Réferéndum e Iniciativa Legislativa.

4 En diversos paises de América Latina en la década de los cincuenta y sesenta, se implementaron programas de desarrollo de las comunidades promocionados por organismos intemacionales: Cepal y Allanza para el progreso. 
nuo crecimiento de las organizaciones vecinales, ha introducido en los sistemas polfticos la posibilidad de ampliación de la participación democrática. Pero, esto dependerá, tanto, de la organización comunal y vecinal que a través de su formación y educación puedan lograr su autonomía e incidir en la esfera polltica, como, de que efectivamente se produzcan cambios en los sistemas politicos que permitan la participación ciudadana.

En Colombia, se distinguen tres etapas, signadas por una confusa y compleja legislación (Leal y Dávila, 1994; 219 230). Entro 1958-1968 la organización comunal cumplió una primera etapa de carácter embrionario. A pesar de que se crearon las primeras Juntas, éstas no tenlan todavía un carácter definitivo como organismos de tipo vecinal barrial o veredal. Su adscripción ínicial fue al Ministerio de Educación (1959), siendo trasladada esta responsabilidad posteriormente al Ministerio de Goblerno (1960). A medida que aumentaba el número de las Juntas se hizo indispensable precisar las normas que las rigieran; por primera vez, se le asignó el papel de promotoras y ejecutoras de obras, lo cual sirvió de base para definir la naturaleza de tales organizaciones.

La segunda etapa entre 19681976, on la cual se consolidan los organismos comunales y se logra la inserción definitiva dentro del sistema politico colombiano. Se promulga el Decreto 3159 a través del cual se intenta manejar o centralizar y ordenar administrativamente este programa bajo la dependencia del Ministerio de Gobierno. El Decreto 2070 reglamentario del antes citado, desarrollo la primera legislación concreta con respecto a las Juntas. Precisó su carácter vecinal, barrial o de corregimiento y señalo los requisitos para que aquéllas adquirieran aquellas personaría jurídica. Esta reglamentación era rígida en los aspectos señalados, pero dejaba campo abierto a las actividades que prioritariamente las Juntas de Acción Comunal y Vecinal debian desempeñar como ejecutoras de obras. Se convirtieron en organizaciones receptoras de recursos y auxilios intermediados por jefes políticos y regionales quienes les utilizaron como mecanismos para la tramitación de las prácticas clientelistas.

En tercera etapa, comprendida entre 1976-1994, se produce la extensión y crecimiento del movimiento comunal dentro de los parámetros antes señalados. Cabe observar que durante este perlodo; el número de Juntas se incremento a 32.000 en todo el país. Este crecimiento condujo a la creación de los Departamentos Administrativos de Acción Comunal como fórmula descentralizadora de los entes encargados del control de la acción comunal. El Decreto 1930 (1979), derogó el anterior y se convintió en el eje normativo de la acción comunal, su principal aporte fue definir el carácter de las Juntas de acción comunal como corporaciones civicas, ejecutoras de politicas públicas. El Decreto 2851 (1984), señaló limites de funcionamiento de Asociaciones Municipales de Juntas de Acción Comunal. No obstante esta legislación no implicaba cambios sustanciales en cuanto a la naturaleza y funcionamiento de la acción municipal.

El Decreto 300 (1987) nuevamente 
ordena todo lo referente a la acción comunal e incorpora nociones como gestión local, integración y organización comunitaria. Coincide con los procesos de descentralización y reforma municipal que impulsa el gobierno de Virgilio Barco, en el marco de la cual se consideró, además que las Juntas podian ejecutar actividades de economía social y autogestión.

Los acontecimientos posteriores evidencian un panorama contradictorio. Si bien es cierto que la organización comunitaria se esforzaba por lograr autonomía y trataba de apoyarse en espacios políticos abiertos con la reforma municipal y la descentralización, una mayoría de Juntas segulan bajo el control institucional y cooptadas, mediatizadas por las redes clientelares partidistas (Leal y Dávila, 1994: 219-230).

Es importante señalar que la Constitución Colombiana de 1991 en el Título $X I$ relativo a la participación democrática de las organizaciones no gubernamentales, incluye además de las organizaciones de servicios profesionales sin ánimo de lucro dedicadas a desarrollar actividades de interés público (fundaciones, asociaciones y corporaciones), a las organizaciones sociales y comunitarias (Art. $\left.87^{\circ}\right)$.

Con esta ley se pretende desmontar la monopolización del Estado sobre las políticas públicas, particularmente en lo atinente a la prestación de servicios sociales y domiciliarios. Esta responsabilidad debe ser compartida con la sociedad civil, o sea con el sector privado y las organizaciones sociales y comunitarias.

Para lograr esto, la ley se propone desarrollar dos aspectos: primero, regla- mentar los mecanismos a través de los cuales la sociedad podrá ejecutar políticas públicas; segundo, la ley pretende garantizar a todos lo actores de la sociedad civil, particularmente a los sectores sociales más necesitados y a las agencias profesionales que con ellos trabajan la competencia en el mercado de servicios y de ejecución de políticas públicas. Esto implica adoptar una forma empresarial con capacidad de contratación con el Estado, que no tienen, especificamente las organizaciones sociales y comunitarias (Ochoa y Restrepo, 1993).

Se observa en el vigente texto constitucional Colombiano y en las Leyes que de éste se derivan en relación con la organización comunitaria, que se afianza el carácter de las Juntas como empresas corporativas que hablan venido conformándose a través de las etapas señaladas anteriormente.

A pesar de que esta legislación declara formalmente que pretende establecer una democracia participativa, en realidad, evidencia una marcada tendencla neoliberal que se concreta al otorgarle a las organizaciones comunales, el papel de empresas corporativas, fundamentalmente ejecutoras de obras, es decir, se trasladan o privatizan algunas funciones en relación con prestación de servicios públicos. No se les otorga participación decisional sobre los asuntos comunitarios $y$ en consecuencia se interfiere en su autonomla. Se observa a lo largo del proceso de institucionalización de las organizaciones comunales, que las blites pollticas mantienen la mediatización o cooptación de estas organizaciones, a través de la orientación que se ha dado a esta le- 
gislación con miras a conservar su poder politico (concentración de poder).

En Venezuela, con sus particulares condiciones socio-politicas y con una legislación menos profusa, igualmente se observan tres etapas en la institucionalización comunitaria y vecinal (Leal, 1991: 168-185).

La primera etapa $1958-1978$ de formación o conformación vecinal, coincide con el inicio de los gobiemos democráticos. Se establecieron programas para el Desarrollo de la Comunidad con miras a organizar a los ciudadanos e incorporarlos en la búsqueda de solución de sus problemas. Esto dio origen a las Juntas de Desarrollo de la Comunidad o Juntas Pro-Mejoras. En el caso de los barrios, estas Juntas se organizaron básicamente, para la consecución y mejoras de los barrios. En las urbanizaciones se crearon las Asociaciones de Propietarios y Residentes, a fin de que tanto los Concejos Municipales como las Compañías Unanizadoras cumplieran con los servicios urbanisticos. Igual que en sistema politico colombiano, en esta etapa no existla una legislación que normatizara a estas organizaciones y el papel que asumieron fueron de carácter reivindicativo de los intereses sociales y como promotores y ejecutoras de obras.

En la segunda etapa 1978-1988 se consolida la organización vecinal a través del reconocimiento institucional de las Asociaciones de Vecinos, mediante la promulgación de la Ley Orgánica de Régimen Municipal (1978) la cual contempla un apartado sobre la Participación de la Comunidad on los asuntos locales y el Reglamento Parcial No. 1 (1979), en don- de se define la regulación de las Asociaciones de Vecinos como expresión organizada de las comunidades y sus relaciones con los diferentes entes del Estado, tanto nacionales, regionales como municipales. Esta ley venía a desarrollar la declaratoria de autonomía de los municipios ya prevista en la Constitución de $1961, y$ algunas regulaciones innovadoras en relación a la participación ciudadana; no obstante, la ley no fue desarrollada o instrumentada en la praxis. El Reglamento vecinal que si fue puesto en vigencia, establecía la forma de obtener la personeria juridica, objetivos; estructura organizativa y funcionamiento de las Asoclaciones de Vecinos. También otorgó excesivos controles a los entes municipales en la determinación de la existencia de las Asociaciones de Vecinos.

A diferencia de la legislación colombiana, las Asociaciones de Vecinos se mantienen como organizaciones de carácter reivindicativo de los intereses comunitarios y de colaboración con el Estado en la prestación de los servicios públicos. Se observa similitud con Colombia en la interferencia que se hace al proceso de institucionalización de estas organizaclones, por los entes municipales y el sistema polftico partidizado, dejando en un segundo plano los procesos de formación o educación de las comunidades en la participación ciudadana.

La tercera etapa 1989-1995 de impulso en la formación de las Asociaciones de Vecinos en todo el territorio nacional coincide con el inicio del proceso de descentralización. Se promulga la Reforma Parcial de la Ley Orgánica de Régimen Municipal (1989) que se propone trasla- 
dar al nivel municipal el ejercicio democrático, a través de una serie de transformaciones en relación a la estructura organizativa y funcionamiento de los Municipios. Adicionalmente a los mecanismo de participación política que establecía la legislación anterior, esta reforma de ley sancionó otros mecanismos de naturaleza diferente entre los cuales se encontró el referéndum municipal. También crea instancias (Juntas Parroquiales) para descentralizar la administración municipal y promover la participación ciudadana. Así mismo, se promulga un nuevo Reglamento Vecinal (1990), se mantiene el carácter reivindicativo y de contraprestación con el Estado en la ejecución de las políticas públicas de las Asociaciones de Vecinos. Se introducen algunos cambios en el proceso de legalización y en la forma de la estructura organizativa. Se trata de fortalecer la autonomía de esas organizaciones al eliminar los controles previos que ejercian los Concejos Municipales para su legalización (determinación de su ámbito territorial). Actualmente se estima que existen en todo el país aproximadamente entre 10.000 a 15.000 Asociaciones de Vecinos.

En esta etapa, el proceso de institucionalización de las Asociaciones de vecinos sigue presentando los mismos obstáculos que en la etapa anterior a pesar de haberse producido cambios en el escenario politico venezolano. Se observa principalmente la interferencia par- tidista (cooptación y mediatización de las asociaciones).

Igual que el proceso colombiano de institucionalización de las organizaciones comunitarias; on el proceso venezolano, también se evidencia en las leyes y reglamentos vecinales una tendencia neoliberal que interfiere en el logro de una democracia más participativa. En efecto; a las Asociaciones de Vecinos se les asigna como principal función la ejecución de las polfticas públicas que se deciden en otros niveles (principalmente en el municipal) diferentes a los de los propias organizaciones vecinales; por ende, no se traslada poder de la sociedad política a la sociedad civil. Se observa que por medio de esta legislación se obstaculiza la autonomia vecinal con lo que se afianzan los mecanismos políticos existentes.

\section{Logros y Obstáculos}

La consolidación y crecimiento de la acción comunal y vecinal en Colombia y Venezuela ha sido continua a lo largo de las tres décadas de su existencia ${ }^{5}$.

En Colombia a medida que se desarrollaba la organización vecinal y se veía su adecuación al sistema político, la intermediación de los partidos en la relación entre Estado y comunidad se convirtió en eje de funcionamiento de estas organizaciones. A ello ayudó la confusa organización institucional. La racionalirealizados por Francisco Leal Buitrago y Andrés Dávila (1994) en Colombla y Nila Leal González $(1991,1996)$ en Venezuela en relación a la Operatividad de la Democracia Participativa. 
dad politico-clientelista explica el funcionamiento de una Oficina de Acción Comunal encargada del control de las Juntas sin recursos y sujetas a tres regimenes administrativos diferentes y simultaneos (nacional, departamental y municipal). El control institucional, si bien se justificaba en tórminos técnicos, obedecía a una fuerza superior determinada por los intereses políticos. La labor técnica de promoción y supervisión ha sido posible gracias a lo que estas organizaciones representan para reproducir el capital electoral.

En Venezuela, la organización vecinal también ha estado sujeta a la mediatización de los partidos en su interrelación con el Estado. Para la supervisión y promoción de las Asociaciones de Vecinos, los Concejos Municipales han creado dependencias administrativas o unidades de desarrollo y promoción comunitaria, oficinas que dependen especfficamente de la Alcaldla, quien proporciona el asesoramiento para la constitución y funcionamiento de estas asociaciones e igualmente asegurar el apoyo electoral.

Si bien la organización de la comunidad genera una dinámica propia en sus actividades, la cual depende de características y condiciones particulares, la forma como han venido siendo cooptadas y mediatizadas, limita las posibilidades de desarrollar manifestaciones autónomas.

Las comunidades organizadas encontraron que la formula prácticamente exclusiva para acceder a la soluciones parciales de sus necesidades materiales era a través de las organizaciones partidistas y con la aceptación de las reglas impuestas por el sistema. Los aspectos relacionados con la capacitación, con el desarrollo de actividades participativas y de conciencia comunitaria quedaron relegados a un plano secundario en ambos sistemas políticos.

Para lograr atención a sus deman. das las Juntas de Acción Comunal y las Asociaciones de Vecinos pueden recurrir a las tres instancias del Estado: nivel local (Alcaldía, Cámara Municipal, Juntas Parroquiales), nivel regional (dependencias de la gobernación, Asambleas), nivel nacional (institutos descentralizados; ministerios, auxilios parlamentarios). En todo caso, siempre están presentes los mecanismos de intermediación partidistas que operan en cada nivel institucional.

La diferencia que existe entre las actividades que realizan las Juntas de Acción Comunal en Colombia y las Asociaciones de Vecinos en Venezuela está en estrecha relación a la naturaleza y carácter asignado a estas organizaciones. Las Juntas de acción comunal funcionan como empresas corporativas que contratan con el Estado como receptoras de recursos y ejecutoras de obras, esto implica un difícil y complejo proceso para la consecución de los recursos, cobro y posterior ejecución de la obra contratada con el Estado, quien en definitiva determina el tipo de obra y el monto asignado, que generalmente es de menor cuantia. Las Juntas solo gestionan no deciden sobre las obras que realizan.

También supone, por un lado, que las Juntas deben cumplir con innumerables requisitos formales y burocráticos, por otro lado, las comunidades requieren 
la asesoria de los promotores para cumplir los trámites, lo cual asegura el control institucional sobre tales organizaciones.

El incremento de requisitos ha servido al mismo tiempo, para que intermediarios politicos -no sin antes el cobro de comisiones- aseguren que los recursos otorgados lleguen a los destinatarios debidos, a cambio de que les den además el beneficio de sus votos.

En Venezuela, las Asociaciones de Vecinos en su carácter de organizaciones reivindicativas de los intereses sociales y comunales, tienen como su actividad fundamental gestionar ante los organismos institucionales, principalmente los municipales se atienda la solución de sus problemas de servicios públicos, vivienda, seguridad personal; y de colaboración con los organismos encargados en la administración de estos servicios, también pueden proponer ante los órganos públicos competentes planes y programas para el mejoramiento de la calidad de vida en el ámbito territorial de la Asociación y del Municipio, señalando prioridades. Las Asociaciones no acceden a la toma de decisiones, solo vigilan y colaboran con el Estado. Esto supone, que las Asociaciones del Vecinos deban realizar una serie de difíciles y complicados trámites burocráticos para que sus demandas sean respondidas por las diferentes instancias municipales (Juntas Parroquiales, Cámara Municipal, Alcaldía, Contraloria Municipal) y otras entidades estatales.

Aqul opera igualmente una red de intermediación politico-partidista, a través de la cual las organizaciones vecinales reciben parcialmente la atención de sus necesidades a cambio de su fidelidad con las organizaciones politicas que controlan el aparato estatal y que son justamente las que viabilizan u obstaculizan en muchas oportunidades estas demandas a conveniencia de sus intereses grupales y sectoriales.

Es de destacar, la semejanza que en Colombia y Venezuela tienen la organización comunal y vecinal en cuanto a que el funcionamiento de estas organizaciones ha dependido y se ha reducido al interés, preocupación y dedicación de las personas elegidas para conformar las juntas directiva en cada perfodo. Existe coincidencia, en que alrededor de esta situación se ha desenvuelto prácticamente lo referente a la participación de la comunidad en ambos paises.

En pocas organizaciones comunales y vecinales se realizan asambleas generales periódicas. Lo común ha sido que la asamblea se reúna solamente para elegir directivos, quedando reducida fundamentalmente las actividades de las organizaciones vecinales y comunales a las reuniones períbdicas de cada junta o mesa directiva. La mayoria de la base comunal no participa efectivamente.

Igualmente es indudable la presencia partidista en las actividades de las juntas directivas, la proyección política de IIderes comunales, la existencla de federaciones y confederaciones vecinales afiliadas a los partidos políticos hace evidente la mediatización entre la actividad de las organizaciones vecinales y las organizaciones politicas.

La participación comunitaria en ambos sistemas politicos es concebida como una participación-gestión que no conducen a una ampliación de la demo- 
cracia; puesto que tanto en Colombia como en Venezuela, esta participación la reducen a funciones meramente de gestión de servicios públicos. Esto es; la participación-gestión es una participación fundamentalmente representativa (mediatizada por los partidos políticos), lo cual se observa tanto en la relación de las organizaciones comunales con los entes municipales donde éstas no tienen una participación directa y decisional; como en el interior de las organizaciones; en donde la base comunitaria no participa y son las Juntas Directivas las encargadas de gestionar la solución de los problemas que concierne a las comunidades.

Es importante destacar que a pesar de que se propicia una participación-gestión en ambos procesos de reforma; igualmente se deja de lado la preparación y formación de la educación participativa; aspecto esto esencial para el desarrollo de las capacidades de gestión de las organizaciones comunales (este aspecto deberá ser abordado en futuras investigaciones dado la importancia que estas organizaciones tienen en los procesos de democratización y desarrollo local).

\section{A manera de conclusión}

A pesar de las particulares diferencias señaladas en relación con la legislación y procesos de organización comunal $y$ vecinal en Colombia y Venezuela, la tendencia central coincidente en ambas reformas polfticas es: reducir la participación comunitaria en participación-gestión la cual no se traduce en una verdadera democratización que implicarla acceder a la toma de decisión y a la extensión del poder de la sociedad política a la sociedad civil.

En este sentido la participación propuesta tiene como norte la privatización de las políticas públicas, esto es, el traslado de las políticas y funciones estatales hacia las comunidades quienes las asumen descargando al Estado de sus responsabilidades. El que estas politicas públicas sean asumidas en su responsabilidad por las Juntas de Acción Comunal (empresas ejecutoras de obras) y por las Asociaciones de Vecinos (gestoras de prestación de senvicios públicos) implica involucrar a los individuos y comunidades organizadas en su ejecución, al mismo tiempo que como usuarias de estos servicios.

Además esta forma de participación exige formas de organización y de representación de intereses según los objetivos institucionales (leyes y reglamentos), desconociendo las formas y prácticas sociales preexistentes. De tal manera, que esta forma de participación (en sus diversos instancias y mecanismos) por un lado, refuerzan y ampllan la dispersión y fragmentación social y por otro lado, reacomoda las relaciones de cooptación e intermediación de la sociedad civil por el Estado y el sistema político.

Las reformas politicas planteadas de acercamiento del Estado al ciudadano común a través de diversos mecanismos y modalidades de participación parecen adquirir más un enfasis procedimental que minimiza los aspectos socio-políticos y económicos. Se privilegia una relación pragmática signada por los problemas concretos que no trascienden lo inmedia- 
to y por ende de poca incidencia en lo regional y nacional.

Se preconiza o recomienda como deseable una ciudadanla organizada que avance hacia niveles superiores de gestión y desarrollo acordes con las exigencias del Estado moderno. Para ello la reforma del Estado dice estar abocada a la creación de la sociedad civil desde arriba, desde las iniciativas públicas. No obstante; en la práctica se están reproduciendo relaciones que mantienen la concentración de poder político en manos de ciertos grupos y organizaciones politicas, ahora encubiertos por formas, mecanismos y procedimientos que definen la participación comunitaria sólo a niveles de participación-gestión; sin incidencia directa y politica, dejando de lado, justamente aspectos esenciales (formación y educación ciudadana), que posibilitan el desarrollo de su autonomia y de capacidad de gestión.

La Reforma Política planteada en Colombia y Venezuela debe corregirse y profundizarse: se deben redefinir estrategias que contribuyan a lograr un equilibrio del poder entre los diversos actores que integran los sistemas politicos; estas es. trategias, se deben reflejar en leyes $y$ reglamentos eficaces, es decir, que realmente posibiliten actores, instancias $y$ mecanismos que propicien la redemocratización que exige la dinámica sociopolitica de ambos sistemas politicos.

\section{Referenclas Blbllográflcas}

Alvarez de Bozo, Míram y Morales, Eduviges (1993). Partlclpación de la Comuntdad en la Prestaclón de los Servlclos Públlcos. Caso Municiplo Mara- caibo, en Cuestiones Polfticas No. $9^{\circ}$. Organo divulgativo del CIEPA. FCJP. LUZ. Maracaibo-Venezuela.

Asamblea Nacional Constituyente de la República de Colombia. (1994). Constltuclón Politlca de Colombla. Reimpresión de Gaceta Oticlal.

Clfuentes Noyes, Ariel; Ochoa, Doris y Restrepo, Dario (1994). Dlez afios de Descentralización. Resultados y Perspectlvas. Edit. Presencla. Bogotá.

Comisión Presidencial para la Reforma del Estado (1988). La Roforma del Eetado. Proyecto de Reforma Integral del Estado. Tomo I. Caracas.

Congreso de la República de Venezuela (1961). Constitución de la Repúbilca de Venezuela. Gaceta Oficial de Venezuela. No. 662.

Congreso de la República de Venezuela (1989). Ley Orgánica de Róglmen Municlpal. Gaceta Oficlal. No. 4.054.

Dlaz Arenas, Pedro Agustin (1993). La Constituclón Polítlca Colomblana (1991). Proceso, Estructura y Contexto. Edlt. Temis S.A. Bogotá.

Leal Bultrago, Ferancisco y Dávila, Andrés (1994). Cllentellsmo. El Sistema PoIitico y su Expresión Reglonal. Instituto de Estudios Pollticos y Relaciones Intemacionales. Bogotá.

Leal González, Nila (1991). Comunidad, Particlpación y Democracia en Venezuela. El Proceso de Institucionalización de las Asociaciones de Vecinos. Mimeo. Caracas.

Leal González, Nila (1996). Participación Cludadana y Democracla. Proyecto de investigación. LUZ-CONDES Maracalbo. Venezuela. Ochoa, Dorls y Restrepo, Darlo (1993). El Estado del Arte de la Descentrallzación Politica y de la Oerta Pública en Participación Cluda* dana y Comunitaria on las Nuevas Politicas. Bogotá. 